\title{
The Algebraic Structure of Cohomological Field Theory
}

\author{
Danny Birmingham ${ }^{1}$ \\ CERN, Theory Division, CH-1211, Geneva 23, Switzerland \\ Mark Rakowski ${ }^{2}$ \\ Institut für Physik, Johannes-Gutenberg-Universität, \\ Staudinger Weg 7, D-6500 Mainz, Germany
}

\begin{abstract}
The algebraic foundation of cohomological field theory is presented. It is shown that these theories are based upon realizations of an algebra which contains operators for both BRST and vector supersymmetry. Through a localization of this algebra, we construct a theory of cohomological gravity in arbitrary dimensions. The observables in the theory are polynomial, but generally non-local operators, and have a natural interpretation in terms of a universal bundle for gravity. As such, their correlation functions correspond to cohomology classes on moduli spaces of Riemannian connections. In this uniformization approach, different moduli spaces are obtained by introducing curvature singularities on codimension two submanifolds via a puncture operator. This puncture operator is constructed from a naturally occurring differential form of co-degree two in the theory, and we are led to speculate on connections between this continuum quantum field theory, and the discrete Regge calculus.
\end{abstract}

Lectures given at the XXVIII Karpacz Winter School, Poland

CERN-TH. 6446/92

April 1992

\footnotetext{
${ }^{1}$ Supported by "Commission des Communautés Européennes" (DG XII-CCR)

Email: Dannyb@cernvm.bitnet

${ }^{2}$ Supported by Bundesministerium für Forschung Technologie

Email: Rakowski@vipmzt.physik.uni-mainz.de
} 



\section{Introduction}

In [1]-[4], it was shown that a certain vector supersymmetry algebra was powerful enough to supply a foundation for both cohomological gauge and gravity theories; the gauge theories could be based on a theory of global vector supersymmetry, while local vector supersymmetry was the essential ingredient for gravity. Although a formulation of cohomological gauge theory was already known [5], an equally satisfactory treatment of gravity was elusive, for various attempts see [6]-[16]. In particular, a set of observables for gauge theory was available [5], and their geometric significance in terms of the universal bundle of Atiyah and Singer [17] was understood [18, 19, 20, 21]. Moreover, it was clear that the observables corresponded to cohomology classes on moduli spaces of gauge connections.

It was observed in [1] that the underlying algebra of cohomological gauge theory contained a global vector supersymmetry operator, in addition to the known BRST symmetry [5]. This led to an algebraic understanding of the descent equations defining the observables, and indicated the generalization to a theory of cohomological gravity. By looking for realizations of a local vector supersymmetry algebra, a multiplet was obtained that was generic to all dimensions [2]. This multiplet does not contain an independent spin connection, and one should not regard this formulation as a "gauge theory" approach to topological gravity. The construction of observables is, nevertheless, entirely natural and parallels in many ways the old results for gauge theory. In particular, there is a natural interpretation in terms of the curvature of a universal bundle for gravity. The observables are polynomial, but generally non-local operators. Such a vector supersymmetry was already observed and exploited in dimension two [22].

Here, we first review the necessary details of the bundle of frames approach to superspace [23]. This provides a unified picture for treating realizations of a global, or local, vector supersymmetry algebra. The basic multiplet and symmetries of cohomological gauge theory are shown to be encoded in this superspace formalism by a particular choice of covariant torsion and curvature constraints [1]. The generalization to gravity is then presented, and the minimal multiplet and observables are obtained [2]. We go on to show how these theories naturally lead one to a uniformization approach to various moduli spaces. While the action for these models describes flat Riemannian connections, the theory also contains a differential form of co-degree two which can be used to introduce curvature singularities on codimension two subspaces. These puncture operators are essentially Wilson lines for theories in three dimensions, and generalize to Wilson "surfaces" in four dimensions. The relevant diffeomorphism group in these theories is one which fixes the tangent space on the punctures. This approach, which is entirely suggested by the continuum field theory, hints at a strong link with Regge calculus [24], and we present some speculative remarks in this direction. The uniformization approach to cohomological gauge theory is also briefly discussed.

\section{Bundle of Frames on Superspace}

We will be interested in constructing realizations of a certain supersymmetry algebra, and here we will appeal to superspace techniques. The advantage of this approach is that supersymmetry transformations can then be interpreted as general coordinate transformations of an underlying supermanifold. The case at hand dictates that our superspace be locally parametrized by the coordinates $z^{\Lambda}=\left(x^{\mu}, \theta^{\alpha}, \theta\right)$, where $x^{\mu}$ is used to denote the Grassmann even coordinates, while $\theta^{\alpha}$ denotes an equal number of odd coordinates and $\theta$ 
is reserved for an additional odd BRST coordinate. Notice that we use lower case Greek indices from the beginning of the alphabet to label the odd coordinates, while those from the middle label the even directions; a capital Greek index denotes generically any of the coordinates.

Over a supermanifold, we can consider a bundle of orthonormal frames by analogy with the familiar construction in conventional geometry [23]. We let $h^{A_{1}}=d z^{\Lambda} h_{\Lambda}^{A_{1}}$ denote such a frame which clearly generalizes the notion of vielbein. The structure group of this bundle is the Lorentz group, and while the formal aspects of the theory are independent of the metric signature, we will mainly be interested in the euclidean case of $O(n)$, where $n$ is the number of even coordinates. An index $A_{1}, B_{1}, \ldots$ is used to denote the horizontal directions in the bundle, and objects with these labels transform in definite representations of the structure group,

$$
D_{A_{0}} h^{A_{1}}=f_{A_{0} B_{1}}^{A_{1}} h^{B_{1}},
$$

where $D_{A_{0}}$ is a generator and satisfies the Lie algebra,

$$
\left[D_{A_{0}}, D_{B_{0}}\right]=f_{B_{0} A_{0}}^{C_{0}} D_{C_{0}}
$$

We will use a lower case Latin index from the beginning of the alphabet to denote an odd "flat" direction, and those near the middle $(i, j, k, \ldots)$ for an even direction. Notice also that the $A_{0}$ subscript labels vertical directions in the bundle. As in supergravity, the even labels transform as vectors under the Lorentz group, $f_{A_{0} j}^{i}=f_{[r s] j}^{i}=\frac{1}{2} \delta_{[r}^{i} \delta_{s] j}$. However, instead of the $h^{a}$ transforming as spinors, we will require them to also transform as vectors. This is simply because we seek to realize a Grassmann odd vector symmetry.

We will also need to introduce a spin connection in this bundle, which we denote in local coordinates by $h_{\Lambda}^{A_{0}}$, and form the covariant derivative

$$
D_{\Lambda}=\partial_{\Lambda}-h_{\Lambda}^{A_{0}} D_{A_{0}}
$$

The spin connection, together with the frame fields, allows us to define both curvature and torsion, and in our conventions take the form

$$
\begin{aligned}
{\left[D_{\Lambda}, D_{\Sigma}\right\} } & =-R_{\Lambda \Sigma}^{A_{0}} D_{A_{0}}, \\
T_{\Lambda \Sigma}^{A_{1}} & =D_{\Lambda} h_{\Sigma}^{A_{1}}-(-1)^{\Lambda \Sigma} D_{\Sigma} h_{\Lambda}^{A_{1}} .
\end{aligned}
$$

It is straightforward to incorporate a Yang-Mills connection into this picture and consider a bundle whose structure group is a product of $O(n)$ with a compact group $G$. In this case, the label $A_{0}$ will denote both of the vertical directions, and when we need to specify more precisely, we will use $A_{0}=[r s]$ when referring to a $O(n)$ direction, and $I, J, K, \ldots$ for the group $G$. It is also conventional to denote the spin and Yang-Mills connections respectively by $h_{\Lambda}^{[r s]}=\omega_{\Lambda}^{[r s]}$ and $h_{\Lambda}^{I}=A_{\Lambda}^{I}$, and $R_{\Lambda \Sigma}^{I}=F_{\Lambda \Sigma}^{I}$ for the Yang-Mills curvature.

The supervielbein also allows one to relate objects with curved and flat indices (note that we reserve $\theta$ for a flat BRST index),

$$
\begin{aligned}
D_{\Lambda} & =h_{\Lambda}^{A_{1}} D_{A_{1}}=h_{\Lambda}^{i} D_{i}+h_{\Lambda}^{a} D_{a}+h_{\Lambda}^{\theta} D_{\theta}, \\
R_{\Lambda \Sigma}^{A_{0}} & =(-1)^{B_{1} \Sigma} h_{\Lambda}^{B_{1}} h_{\Sigma}^{C_{1}} R_{B_{1} C_{1}}^{A_{0}}, \\
T_{\Lambda \Sigma}^{A_{1}} & =(-1)^{B_{1} \Sigma} h_{\Lambda}^{B_{1}} h_{\Sigma}^{C_{1}} T_{B_{1} C_{1}}^{A_{1}},
\end{aligned}
$$

and the entire algebraic picture can be cast very compactly as

$$
\left[D_{A}, D_{B}\right\}=F_{B A}^{C} D_{C}
$$


where $F_{B_{1} A_{1}}^{C_{1}}=T_{B_{1} A_{1}}^{C_{1}} F_{B_{1} A_{1}}^{C_{0}}=R_{B_{1} A_{1}}^{C_{0}} F_{B_{0} A_{1}}^{B_{1}}=f_{B_{0} A_{1}}^{B_{1}}$, and so on.

The Bianchi identities follow from the super Jacobi identity which represents the associativity of the differential operators, and with these conventions they take the form

$$
\begin{aligned}
0 & =\sum_{(\text {cyclic })}(-1)^{A_{1} B_{1}}\left[D_{A_{1}} T_{B_{1} C_{1}}^{D_{1}}-T_{C_{1} A_{1}}^{E_{1}} T_{B_{1} E_{1}}^{D_{1}}+R_{C_{1} A_{1}}^{E_{0}} f_{E_{0} B_{1}}^{D_{1}}\right], \\
0 & =\sum_{\text {(cyclic) }}(-1)^{A_{1} B_{1}}\left[D_{A_{1}} R_{B_{1} C_{1}}^{D_{0}}-T_{C_{1} A_{1}}^{E_{1}} R_{B_{1} E_{1}}^{D_{0}}\right] .
\end{aligned}
$$

The sum here is over the three cyclic permutations of the lower indices.

The convenience of this whole approach is really encoded in the transformation properties of the fields we have introduced; everything is based on general coordinate and tangent frame rotations in the superspace. For example, consider a general coordinate transformation on the tangent frame; the components transform simply as

$$
h_{\Lambda}^{\prime A_{1}}\left(z^{\prime}\right)=\frac{\partial z^{\Sigma}}{\partial z^{\prime \Lambda}} h_{\Sigma}^{A_{1}}(z)
$$

In its infinitesimal form, where $z^{\Sigma}=z^{\Sigma}+\epsilon^{\Sigma}$, we have the equally familiar expression

$$
\begin{aligned}
\delta h_{\Lambda}^{A_{1}} & =h_{\Lambda}^{A_{1}}(z)-h_{\Lambda}^{A_{1}}(z) \\
& =\epsilon^{\Sigma} \partial_{\Sigma} h_{\Lambda}^{A_{1}}+\left(\partial_{\Lambda} \epsilon^{\Sigma}\right) h_{\Sigma}^{A_{1}} .
\end{aligned}
$$

It will be convenient to rewrite the transformations of the fields in terms of an equivalent set of parameters. If we define $\epsilon^{A_{1}}=\epsilon^{\Sigma} h_{\Sigma}^{A_{1}}$, then it follows simply from our definitions that the above general coordinate transformation can be written as,

$$
\delta h_{\Lambda}^{A_{1}}=D_{\Lambda} \epsilon^{A_{1}}-h_{\Lambda}^{B_{1}} \epsilon^{C_{1}} T_{B_{1} C_{1}}^{A_{1}}+\epsilon^{\Sigma} \omega_{\Sigma}^{A_{0}} f_{A_{0} B_{1}}^{A_{1}} h_{\Lambda}^{B_{1}} .
$$

Notice that the last term here has the form of a local frame rotation with parameter $\epsilon^{\Sigma} \omega_{\Sigma}^{A_{0}}$. If we now redefine the parameter for local frame rotations by

$$
\epsilon^{A_{0}} \rightarrow \epsilon^{A_{0}}-\epsilon^{\Sigma} \omega_{\Sigma}^{A_{0}}
$$

we can then write the complete transformation properties of the tangent frame as,

$$
\delta h_{\Lambda}^{A_{1}}=D_{\Lambda} \epsilon^{A_{1}}-h_{\Lambda}^{B_{1}} \epsilon^{C} F_{B_{1} C}^{A_{1}} .
$$

The transformation properties of the spin connection follow in a similar way, and we can represent the algebra of all the local symmetries in the theory in the very compact form,

$$
\begin{aligned}
\delta h_{\Lambda}^{A} & =D_{\Lambda} \epsilon^{A}-h_{\Lambda}^{B_{1}} \epsilon^{C} F_{B_{1} C}^{A}, \\
\delta F_{B C}^{A} & =\epsilon^{E} D_{E} F_{B C}^{A} .
\end{aligned}
$$

Note that $A, B, \ldots$ with no subscript denotes either a vertical or horizontal direction.

The gravitino field $h_{\mu}^{a}$ is sometimes more conventionally denoted by $\psi_{\mu}^{a}$. The spin connection of course enters into the algebra (14), however, it is not an independent field as it can generically be expressed in terms of the vielbein and the torsion component $T_{\mu \nu}^{i}$. In our construction of vector supergravity, this torsion component will vanish, and we will effectively be considering Riemannian connections.

The superspace we have constructed here mirrors the standard formulation used in conventional supergravity [25, 26]. Other essentially equivalent approaches can be found in numerous books dealing with supergravity, e.g. [27], and one will be able to quickly reproduce our results in any of these systems. 


\section{Cohomological Gauge Theory}

In cohomological gauge theory, it was known that a certain set of fields, together with their BRST transformations, formed a basis for the theory [5]. A geometrical understanding of these fields finds a natural place within the universal bundle construction of Atiyah and Singer [17]; this was observed by several groups [18, 19, 20, 21]. In addition, it was shown that a vector supersymmetry operator played an important role in these models $[1,3]$. The term vector supersymmetry simply indicates that the infinitesimal parameter in the transformations (or equivalently the operator which generates these transformations) is a Grassmann odd vector. The aim here is to show how these fields, and their transformations, can be encoded in the superconnection formalism. When phrased as a problem in superspace, the task is to find a covariant solution to the Bianchi identities of the previous section. Indeed, we shall find that the basic set of fields furnishes a realization of the global BRST-SUSY algebra.

To begin, let us specify the BRST symmetry transformations; we have [5]

$$
\begin{aligned}
\delta A_{\mu}^{I} & =\epsilon \delta_{\mu}^{i} \psi_{i}^{I}, \\
\delta \psi_{i}^{I} & =\frac{\epsilon}{2} D_{i} \phi^{I}, \\
\delta \phi^{I} & =0 .
\end{aligned}
$$

The statistics and ghost numbers of $\left(A_{\mu}, \psi_{i}, \phi\right)$ are given by $(+,-,+)$ and $(0,1,2)$, respectively. It is an easy task to verify that the corresponding algebra closes up to a Yang-Mills gauge transformation with parameter $\phi$, that is,

$$
\left[\delta_{B R S T}\left(\epsilon_{1}\right), \delta_{B R S T}\left(\epsilon_{2}\right)\right]=\delta_{Y M}\left(\epsilon^{I}=-\epsilon_{1} \epsilon_{2} \phi^{I}\right) .
$$

In addition, we can also define the following vector supersymmetry transformations [1]:

$$
\begin{aligned}
\delta A_{\mu}^{I} & =0 \\
\delta \psi_{i}^{I} & =\epsilon^{a} \delta_{a}^{j} F_{j i}^{I}, \\
\delta \phi^{I} & =2 \epsilon^{a} \delta_{a}^{i} \psi_{i}^{I} .
\end{aligned}
$$

The resulting algebra takes the form:

$$
\begin{aligned}
{\left[\delta_{S U S Y}\left(\epsilon^{a}\right), \delta_{B R S T}(\epsilon)\right] } & =\delta_{D I F F}\left(\epsilon^{i}=\delta_{a}^{i} \epsilon \epsilon^{a}\right), \\
{\left[\delta_{S U S Y}\left(\epsilon_{1}^{a}\right), \delta_{S U S Y}\left(\epsilon_{2}^{b}\right)\right] } & =0 .
\end{aligned}
$$

It is clear at this point that the entire formalism is covariant with respect to the YangMills gauge symmetry. Constructions such as this, where the BRST operator is nilpotent up to a certain gauge transformation, are called "equivariant". Our task is therefore to establish whether this algebra can be embedded in the superconnection framework that was described earlier.

Since the symmetries on hand are of a global nature (both $\epsilon$ and $\epsilon^{a}$ being constant), we initiate our search by demanding that the vielbein describes a flat geometry, $h_{\mu}^{i}=\delta_{\mu}^{i}$. Furthermore, the potential gauge fields for the SUSY and BRST symmetries are taken to be trivial, $h_{\mu}^{a}=0, h_{\mu}^{\theta}=0$. It remains to determine the appropriate set of curvature and torsion constraints. The fundamental torsion constraints are immediately specified 
by the algebra of interest, namely (16) and (18), and we thus find only one non-vanishing component

$$
T_{a \theta}^{i}=\delta_{a}^{i}
$$

This is analogous to the fundamental torsion constraint $T_{a b}^{i}=\gamma_{a b}^{i}$ in standard supersymmetry, where the lower indices here denote spinor directions. Equation (16) also dictates the curvature constraint,

$$
F_{\theta \theta}^{I}=\phi^{I} \text {. }
$$

Finally, re-writing (15) for the Yang-Mills connection in the form (14),

$$
\delta A_{\mu}^{I}=D_{\mu} \epsilon^{I}-h_{\mu}^{i} \epsilon F_{i \theta}^{I}-h_{\mu}^{i} \epsilon^{a} F_{i a}^{I}-h_{\mu}^{j} \epsilon^{i} F_{j i}^{I},
$$

one finds the remaining curvature constraint,

$$
F_{\theta i}^{I}=\psi_{i}^{I}
$$

All other curvature components (apart from $F_{i j}^{I}$ ) are zero.

The consistency of this set can now be verified through an analysis of the Bianchi identities (8). In particular, we have the following non-trivial identities

$$
\begin{aligned}
(\theta i j)^{I}: & =D_{\theta} F_{i j}^{I}+D_{[i} F_{j] \theta}^{I}, \\
(a i j)^{I}: & =D_{a} F_{i j}^{I}, \\
(\theta \theta i)^{I}: & =2 D_{\theta} F_{i \theta}^{I}+D_{i} F_{\theta \theta}^{I}, \\
(\theta \theta a)^{I}: & =D_{a} F_{\theta \theta}^{I}+2 \delta_{a}^{i} F_{i \theta}^{I}, \\
(\theta a i)^{I}: & =D_{a} F_{i \theta}^{I}+\delta_{a}^{j} F_{j i}^{I}, \\
(\theta \theta \theta)^{I}: 0 & =D_{\theta} F_{\theta \theta}^{I} .
\end{aligned}
$$

It is straightforward to check that (23) reproduces the transformations and algebra of the fundamental fields $\left(A_{\mu}, \psi_{i}, \phi\right)$ given above. We have thus succeeded in obtaining a covariant solution to the superconnection formalism.

One of the most important features of cohomological gauge theory is the construction of a family of metric independent observables. Since the algebra above is equivariant with respect to Yang-Mills symmetry, these observables are given by BRST invariant, and Yang-Mills invariant functionals of the minimal multiplet of fields. From (15), we see that the field $\phi$ is itself BRST invariant, and the first observable is formed by taking, for example [5],

$$
W^{(0,4)}=\frac{1}{2} \operatorname{tr} \phi^{2}=\frac{1}{2} \operatorname{tr} F_{\theta \theta}^{2},
$$

where the superscript indicates that it is a zero-form of ghost number four, and the trace is the usual Yang-Mills trace. Given this initial observable, one can construct an entire hierarchy of observables by successive applications of the vector supersymmetry operator $[1,3,28]$. To this end, one notes that the latter connects successive components of curvature in the following manner [1]:

$$
\delta_{S U S Y}: F_{\theta \theta} \rightarrow F_{\theta i} \rightarrow F_{i j} \rightarrow 0
$$

Indeed, as a 1-form with ghost number -1 , it is naturally designed for this purpose. A tower of differential form operators is obtained by repeated application of $\delta_{S U S Y}$ :

$$
W_{i}^{(1,3)}=2 \operatorname{tr} F_{\theta i} F_{\theta \theta},
$$




$$
\begin{aligned}
& W_{i j}^{(2,2)}=4 \operatorname{tr}\left(F_{\theta i} F_{\theta j}+\frac{1}{2} F_{\theta \theta} F_{i j}\right), \\
& W_{i j k}^{(3,1)}=4 \operatorname{tr}\left(F_{\theta i} F_{j k}+F_{\theta j} F_{k i}+F_{\theta k} F_{i j}\right), \\
& W_{i j k l}^{(4,0)}=4 \operatorname{tr}\left(F_{i j} F_{k l}+F_{i k} F_{l j}+F_{i l} F_{j k}\right) .
\end{aligned}
$$

To actually construct the observables, one integrates each of these forms over an appropriate homology cycle on the manifold $M$. The verification that they are BRST invariant, and depend only on the chosen homology class, follows from a set of descent equations. We defer a discussion of these points to section 5, where both the gauge and gravity cases can be treated simultaneously.

As we have seen, there are three non-vanishing components of curvature in the superspace $\left(x^{\mu}, \theta^{\alpha}, \theta\right)$, namely, $\left(F_{\theta \theta}, F_{\theta i}, F_{i j}\right)$. Clearly, the sum of these three pieces represents the entire curvature, $F$, of superspace, and hence one can form a characteristic class $\operatorname{Tr} F^{2}$, for example. By construction, the Bianchi identities ensure that this object is a closed 4-form. Furthermore, one sees that the sum of (24) and (26) is equal to this class. In this way, one obtains a geometrical understanding of the fields and observables in cohomological gauge theory. Indeed, the superspace curvature is precisely the curvature of the universal bundle of Atiyah and Singer, whose construction we now briefly review.

Given a principal bundle $P$, with structure group $G$, over a compact smooth manifold $M$, one can consider the affine space $\mathcal{A}$ of all connections on $P$. This space is acted on by the group $\mathcal{G}$ of pointed gauge transformations, and leads one to the principal $\mathcal{G}$-bundle $P \times \mathcal{A}$ over $Q=(P \times \mathcal{A}) / \mathcal{G}$. Since $G$ acts freely on the base $Q$ of this bundle, the following triplet

$$
\mathcal{Q}=((P \times \mathcal{A}) / \mathcal{G}, G, Q / G=M \times \mathcal{A} / \mathcal{G}),
$$

defines the so-called 'universal' $G$-bundle over the base space $M \times \mathcal{A} / \mathcal{G}$.

Now, differential forms on $M \times \mathcal{A} / \mathcal{G}$ carry a natural bi-grading, a $(p, q)$-form referring to a $p$-form on $M$ and a $q$-form on $\mathcal{A} / \mathcal{G}$. Previously, we have used the term 'ghost number' for the $q$ degree. The connection on the universal bundle can, therefore, locally be written as

$$
\hat{A}=A_{(1,0)}+A_{(0,1)} .
$$

Similarly, the universal curvature, being a 2-form, decomposes as

$$
\begin{aligned}
\mathcal{F} & =\left(d+\delta_{B R S T}\right) \hat{A}+\frac{1}{2}[\hat{A}, \hat{A}] \\
& =\mathcal{F}_{(2,0)}+\mathcal{F}_{(1,1)}+\mathcal{F}_{(0,2)},
\end{aligned}
$$

where $d$ is the usual exterior derivative on $M$, and $\delta_{B R S T}$ its $(0,1)$ counterpart. It is clear that the components of the universal curvature have the correct degree in order to allow them to be identified with the $\left(F_{i j}, F_{\theta i}, F_{\theta \theta}\right)$ components of the superspace curvature. In addition, the covariant solution of the superspace Bianchi identities dictates precisely the three non-vanishing components of the universal curvature. As a final remark, we note that in principle one can consider higher characteristic classes, although for technical reasons they have thus far not been used in cohomological gauge theory.

By construction, the parameter in the vector supersymmetry transformations is a constant vector. Therefore, if we wish to define this as a symmetry of the theory on a general manifold, we encounter the integrability constraint: $\left[D_{\mu}, D_{\nu}\right] \epsilon_{\lambda}=R_{\mu \nu \lambda \sigma} \epsilon^{\sigma}=0$. Solutions to this constraint are provided in cases where either the Riemann curvature vanishes, or has zero modes. The (rigid) validity of the vector supersymmetry is thus restricted to certain manifolds. The natural way to overcome this integrability constraint 
is to localize the vector supersymmetry. Our aim will be to first construct a theory of vector supergravity, and we shall then find that the coupling to the gauge system follows unhindered.

\section{Cohomological Gravity}

In order to construct a theory of vector supergravity, one begins by allowing the vielbein $h_{\mu}^{i}$, and its gravitino $h_{\mu}^{a}=\psi_{\mu}^{a}$, to be arbitrary; in the present analysis, we are maintaining the triviality of the BRST gauge field $\left(h_{\mu}^{\theta}=0\right)$. Including the gravitino corresponds to localizing the vector supersymmetry, and hence leads one to a theory of vector supergravity. The crucial step is then to decide on an appropriate set of covariant torsion constraints. The most promising road to take, and the one which yields the present solution, is to discard all extra torsion components which have negative or zero ghost number. We do, however, retain the $T_{a \theta}^{i}$ piece, in order to retrieve the global result in the limit of flat vielbein and vanishing gravitino. We should stress that the specification of these torsion constraints dictates the additional fields which are required to complete the vector supergravity multiplet. Unlike the case of gauge theory, here we do not know the basic multiplet, and some nimble footwork is needed in order to obtain a consistent field content. As it turns out, it is also possible to set to zero the five components $T_{\theta \theta}^{i}, T_{\theta \theta}^{a}$, $T_{b \theta}^{a}, T_{j \theta}^{i}$, and $T_{\theta \theta}^{\theta}$, and we will do so in the following. In [2], another non-minimal solution to the Bianchi identities was presented which incorporated $T_{\theta \theta}^{i}$ and $T_{\theta \theta}^{a}$ as independent fields, and one can consider this larger set of fields as a coupling of a new multiplet to the minimal multiplet. Since the observables in this extended multiplet are unclear, we will not consider it here.

Without further ado, let us present the non-vanishing torsion components [2]:

$$
T_{a \theta}^{i}=\delta_{a}^{i}, T_{i \theta}^{a}=\frac{1}{2} \delta^{a j} \phi_{i j}, T_{i j}^{a},
$$

where $\phi_{i j}$ is antisymmetric in its indices, that is, it corresponds to a scalar field in the adjoint representation of the Lorentz group. It should be noted that the final component in this set, $T_{i j}^{a}$, is not an independent field, and can be expressed in terms of derivatives of the gravitino. A slight note of warning is required in the use of (30); the $i$ and $a$ indices label different spaces and thus caution must be exercised accordingly.

At this juncture, one must simply analyze the resulting set of Bianchi identities. One can regard this formalism as providing a bootstrap approach to obtaining a solution. Given the above torsion constraints, one examines each of the identities in turn, and checks for consistency. The non-trivial identities are:

$$
\begin{aligned}
& (a b i)^{j}: 0=R_{a b}{ }^{i}{ }_{j}, \\
& (a \theta i)^{j}: 0=R_{a \theta}{ }^{j}{ }_{j}, \\
& (a i j)^{k}: 0=R_{a\left[{ }^{k}{ }^{k}\right]}, \\
& (\theta i j)^{k}: 0=R_{\theta\left[{ }^{k}{ }^{k}{ }_{j}-T_{i j}^{a} \delta_{a}^{k},\right.}, \\
& (\theta \theta i)^{j}: 0=2 T_{i \theta}^{a} \delta_{a}^{j}+R_{\theta \theta}{ }^{j}{ }_{i}, \\
& (\theta \theta a)^{b}: 0=\delta_{i}^{b} \delta_{a}^{j} R_{\theta \theta}{ }^{i}{ }_{j}+2 T_{i \theta}^{b} \delta_{a}^{i}, \\
& (\theta \theta i)^{a}: 0=D_{\theta} T_{i \theta}^{a}, \\
& (a \theta i)^{b}: 0=D_{a} T_{i \theta}^{b}+\delta_{j}^{b} \delta_{a}^{k} R_{\theta i}{ }_{k}{ }_{k}-\delta_{a}^{j} T_{i j}^{b}, \\
& (\theta i j)^{a}: 0=D_{\theta} T_{i j}^{a}+D_{[i} T_{j] \theta}^{a},
\end{aligned}
$$




$$
(a i j)^{b}: 0=D_{a} T_{i j}^{b}+\delta_{k}^{b} \delta_{a}^{m} R_{i j}{ }^{k}{ }_{m}
$$

In addition, one finds the non-zero components of curvature (besides $R_{i j}{ }^{r s}$ ) to be:

$$
R_{\theta \theta}{ }^{r s}=\phi^{r s}, R_{\theta i}{ }^{r s}=\frac{1}{2}\left(\delta_{i a} T_{j k}^{a}-\delta_{a[j} T_{k] i}^{a}\right) \delta^{j r} \delta^{k s} .
$$

The transformations of the minimal multiplet are given by

$$
\begin{aligned}
\delta h_{\mu}^{i} & =\epsilon \psi_{\mu}^{a} \delta_{a}^{i}, \\
\delta \psi_{\mu}^{a} & =D_{\mu} \epsilon^{a}-\epsilon h_{\mu}^{i} T_{i \theta}^{a}, \\
\delta \phi^{r s} & =2 \epsilon^{a} \delta_{a}^{i} R_{\theta i}{ }^{r s} .
\end{aligned}
$$

Although the torsion and curvature components $T_{i j}^{a}, R_{\theta i}{ }^{r s}$, and $R_{i j}{ }^{r s}$ are not independent fields, their transformations are simple expressions, and we record them here:

$$
\begin{aligned}
\delta T_{i j}^{a} & =-\frac{\epsilon}{2} D_{[i} \phi_{j] k} \delta^{k a}-\epsilon^{b} R_{i j}{ }^{r s} \delta_{r}^{a} \delta_{s b}, \\
\delta R_{\theta i}{ }^{r s} & =\frac{\epsilon}{2} D_{i} \phi^{r s}-\epsilon^{a} \delta_{a}^{j} R_{i j}{ }^{r s}, \\
\delta R_{i j}{ }^{r s} & =-\epsilon D_{[i} R_{j] \theta}{ }^{r s},
\end{aligned}
$$

where the latter two are obtained from the $(\theta \theta i)^{r s},(a \theta i)^{r s},(\theta i j)^{r s},(a i j)^{r s}$ identities in (8). Finally, one can read off the transformation of the spin connection from (14):

$$
\delta \omega_{\mu}^{r s}=-\epsilon h_{\mu}^{i} R_{i \theta}^{r s} .
$$

One can now check that the above multiplet of fields $\left(h_{\mu}^{i}, \psi_{\mu}^{a}, \phi^{r s}\right)$ provides a representation for a BRST-SUSY algebra, with the following closure properties:

$$
\begin{aligned}
{\left[\delta_{S U S Y}\left(\epsilon^{a}\right), \delta_{B R S T}(\epsilon)\right] } & =\delta_{D I F F}\left(\epsilon^{i}=\delta_{a}^{i} \epsilon \epsilon^{a}\right), \\
{\left[\delta_{B R S T}\left(\epsilon_{1}\right), \delta_{B R S T}\left(\epsilon_{2}\right)\right] } & =\delta_{\text {LORENTZ }}\left(\epsilon^{r s}=-\epsilon_{1} \epsilon_{2} \phi^{r s}\right), \\
{\left[\delta_{S U S Y}\left(\epsilon_{1}^{a}\right), \delta_{S U S Y}\left(\epsilon_{2}^{b}\right)\right] } & =0 .
\end{aligned}
$$

In the above, we have only listed the essential part of the algebra; however, given the full set of torsion and curvature constraints, we can read off the complete algebra from (7). In the larger non-minimal multiplet that we discussed earlier, one finds that the BRST-BRST commutator also contains a diffeomorphism and a local supersymmetry transformation on the right hand side, and this ruins the simple observable structure of the present theory.

We have thus succeeded in constructing a multiplet for vector supergravity. Since the solution involves a localization of a vector supersymmetry, one is naturally led to include a vector gravitino $\psi_{\mu}^{a}$, which then appears as the BRST topological shift partner for the vielbein. We are not treating the spin connection as an independent field; it can be written in terms of derivatives of the vielbein and its inverse in the ususal way by solving $D_{[\mu} h_{\nu]}^{i}=0$ for $\omega_{\mu}^{r s}$. Similarly, the BRST shift partner to the spin connection, $R_{\theta \mu}$, is not independent and represents a deformation of the spin connection induced from a deformation in the vielbein. It is straightforward to consider the coupling of the above gravity multiplet to the gauge multiplet of the previous section, thereby taking the structure group to be a direct product of the Lorentz group and the compact group $G$. By considering the coupled Bianchi identities, now allowing the index $A_{0}$ to run over $[r s]$ and $I$, one finds that the transformations of the gauge multiplet are unaltered. The 
only change in the algebra is that one adds the term $\delta_{Y M}\left(\epsilon^{I}=-\epsilon_{1} \epsilon_{2} \phi^{I}\right)$ to the above commutator of two BRST transformations. In performing this coupling, one overcomes the integrability constraint which appeared in the gauge theory case.

In the present theory, we again notice that we have a BRST invariant field $\phi^{r s}$; hence, we can form the observable

$$
W^{(0,4)}=\frac{1}{2} \operatorname{tr} \phi^{2}=\frac{1}{2} \operatorname{tr} R_{\theta \theta}^{2},
$$

where the superscript indicates that it is a zero-form of ghost number four, and the trace represents an $O(n)$ trace. In the case of $2 \mathrm{~d}$ gravity, higher powers of $\phi$ do appear, but for convenience, we restrict our attention to the above example. As for the case of gauge theory, the vector supersymmetry operator connects successive components of curvature:

$$
\delta_{S U S Y}: R_{\theta \theta} \rightarrow R_{\theta i} \rightarrow R_{i j} \rightarrow 0
$$

and we arrive at the following assembly of differential forms:

$$
\begin{aligned}
& W_{i}^{(1,3)}=2 \operatorname{tr} R_{\theta i} R_{\theta \theta}, \\
& W_{i j}^{(2,2)}=4 \operatorname{tr}\left(R_{\theta i} R_{\theta j}+\frac{1}{2} R_{\theta \theta} R_{i j}\right), \\
& W_{i j k}^{(3,1)}=4 \operatorname{tr}\left(R_{\theta i} R_{j k}+R_{\theta j} R_{k i}+R_{\theta k} R_{i j}\right), \\
& W_{i j k l}^{(4,0)}=4 \operatorname{tr}\left(R_{i j} R_{k l}+R_{i k} R_{l j}+R_{i l} R_{j k}\right) .
\end{aligned}
$$

It is straightforward to re-write these expressions with either curved indices, or in differential form notation.

As before, one can construct a characteristic class by forming $\operatorname{tr} R^{2}$, where $R$ represents the entire curvature of superspace, which in the present case consists of three non-vanishing components, $R_{\theta \theta}, R_{\theta i}, R_{i j}$. By construction, the Bianchi identities ensure that this object is a closed 4-form. In [17] (see also [29]), the extension of the universal bundle program to the space of metrics was outlined, and the curvature of the superspace above represents the curvature of this universal bundle. Finally, let us remark that an alternative set of observables presents itself in four dimensions. Using the $S O(4)$ invariant tensor $\epsilon_{m n r s}$, we can form the $S O(4)$ invariant operator

$$
W^{(0,4)}=\frac{1}{2} \epsilon_{m n r s} \phi^{m n} \phi^{r s} .
$$

Proceeding as before, one generates a new hierarchy of operators, with the top form being given by the Euler class, $\epsilon^{i j k l} R_{i j} R_{k l}$.

\section{Observables Hierarchies from Vector Supersym- metry}

It has been known for some time that BRST invariance plays an important role in topological field theory $[18,30,31,19]$; indeed, the observables in cohomological models correspond to BRST cohomology classes, i.e., operators which are BRST-closed, modulo those which are BRST-exact [5]. The observables are defined via a set of operators $W^{(i)}(i=0, \ldots, n)$, which satisfy the following descent equations:

$$
Q_{B R S T} W^{(0)}=0
$$




$$
\begin{aligned}
Q_{B R S T} W^{(1)} & =d W^{(0)}, \\
& \vdots \\
Q_{B R S T} W^{(n)} & =d W^{(n-1)}, \\
0 & =d W^{(n)},
\end{aligned}
$$

where $\delta_{B R S T}=\epsilon Q_{B R S T}$. Here, $i$ denotes the differential form degree, and $d$ is the exterior derivative on $M$; we shall omit the ghost number labelling on the operators. As a consequence of these defining relations, one can now construct a set of observables by integrating each of the operators $W^{(i)}$ over an appropriate homology cycle $\gamma_{i}$, on $M$. Defining

$$
W\left(\gamma_{i}\right)=\int_{\gamma_{i}} W^{(i)}
$$

we see that it is BRST invariant as a result of (41):

$$
Q_{B R S T} W\left(\gamma_{i}\right)=\int_{\gamma_{i}} d W^{(i-1)}=\int_{\partial \gamma_{i}} W^{(i-1)}=0
$$

Furthermore, a simple check reveals that each of these observables depends only on the homology class of $\gamma_{i}$. Integrating over a boundary, we find

$$
W\left(\partial \gamma_{(i+1)}\right)=\int_{\partial \gamma_{(i+1)}} W^{(i)}=\int_{\gamma_{(i+1)}} d W^{(i)}=Q_{B R S T} \int_{\gamma_{(i+1)}} W^{(i+1)}
$$

and hence this object is BRST trivial. In practice, the above hierarchy of equations is initiated by choosing a BRST invariant operator $W^{(0)}$. However, one may wonder whether the ensuing relations in this hierarchy are merely fortuitous, or whether they are a result of some more fundamental property of cohomological field theory.

Let us begin by recalling the essential relations in the BRST-SUSY algebra:

$$
\begin{gathered}
\left\{D_{\theta}, D_{a}\right\}=\delta_{a}^{i} D_{i}, \\
{\left[D_{a}, D_{i}\right]=0,} \\
\left\{D_{a}, D_{b}\right\}=0, \\
\left\{D_{\theta}, D_{\theta}\right\}=R_{\theta \theta}^{C_{0}} D_{C_{0}} .
\end{gathered}
$$

Consider a BRST invariant object $W^{(0)} ; D_{\theta} W^{(0)}=0$, which is also covariant with respect to $O(n)$ rotations, and gauge transformations. The superscript indicates the differential form degree; however, in the following we shall present our results in terms of components. We define

$$
W_{a}^{(1)}=D_{a} W^{(0)}, W_{i}^{(1)}=\delta_{i}^{a} W_{a}^{(1)} .
$$

If we now act on $W^{(0)}$ with $(45)$ we obtain the following result:

$$
D_{\theta} W_{i}^{(1)}=D_{i} W^{(0)}
$$

It is immediately evident that such a relation is precisely the second step in the hierarchy of (41). The reason the covariant derivative $D_{i}$ appears, rather than the exterior derivative as in (41), is simply because our present discussion is more general. We are first considering covariant objects $W^{(i)}$; for the purposes of constructing observables one then specializes to objects which are invariant with respect to $O(n)$ rotations, and Yang-Mills 
transformations. We thus conclude that such a pattern is not simply a twist of good fortune, but is a direct consequence of the underlying vector supersymmetry algebra [3]. This analysis can be continued, and one verifies that the construction of the entire hierarchy (41) is based solely on the three relations (45)-(47). The $k$ th level is obtained by defining

$$
\begin{aligned}
W_{a_{1} \cdots a_{k}}^{(k)} & =D_{a_{1}} W_{a_{2} \cdots a_{k}}^{(k-1)}, \\
W_{i_{1} \cdots i_{k}}^{(k)} & =\delta_{i_{1}}^{a_{1}} \cdots \delta_{i_{k}}^{a_{k}} W_{a_{1} \cdots a_{k}}^{(k)},
\end{aligned}
$$

and using (45)-(47) one finds:

$$
D_{\theta} W_{i_{1} \cdots i_{k}}^{(k)}=\frac{1}{(k-1) !} D_{\left[i_{1}\right.} W_{\left.i_{2} \cdots i_{k}\right]}^{(k-1)} .
$$

For the purpose of illumination, we have chosen to endow the objects $W$ with flat indices. It is quite straightforward to adopt a curved index notation, or write these objects as differential forms. For example, let us define

$$
W_{\mu}^{(1)}=h_{\mu}^{i} W_{i}^{(1)} .
$$

A little extra care is needed here as the operators $D_{\theta}, D_{a}$ do not represent the symmetries when acting on non-covariant objects such as the vielbein $h_{\mu}^{i}$. In this case, one uses the relation (36), and acting on $W^{(0)}$, one finds

$$
Q_{B R S T} W_{\mu}^{(1)}=D_{\mu} W^{(0)}=\left(\partial_{\mu}-\omega_{\mu}^{[r s]} D_{r s}-A_{\mu}^{I} D_{I}\right) W^{(0)} .
$$

Similarly, the general result is given by

$$
Q_{B R S T} W_{\mu_{1} \cdots \mu_{k}}^{(k)}=\frac{1}{(k-1) !} D_{\left[\mu_{1}\right.} W_{\left.\mu_{2} \cdots \mu_{k}\right]}^{(k-1)}
$$

The above derivation of the descent equations which define a cohomological field theory clearly demonstrates that they have their origin in the vector supersymmetry algebra. The knowledge that BRST plays a role in these theories is not a sufficient guarantee for this structure. As we have seen, the BRST operator is nilpotent up to local $O(n)$ rotations, and Yang-Mills gauge transformations. In order to construct an observable, one must therefore ensure that the objects $W$ are invariant with respect to these local symmetries. In practice, this simply involves taking an appropriate group trace.

\section{Field Theoretic Realization of a Universal Bundle for Gravity}

In order to construct a quantum action $S_{q}$, we can proceed in the usual way and take the Lagrangian to be BRST exact [5], namely

$$
S_{q}=\int Q_{B R S T} V
$$

where $V$ is a Lorentz invariant functional which comprises a sum of terms of the form Antighost (Gauge Fixing Condition). This action is evidently invariant under BRST, since, from (36), the BRST operator closes up to a Lorentz rotation. It is at this point 
that we must decide on a moduli space of interest, and the specification of this space corresponds to one of the gauge fixing conditions, which we shall refer to as the topological gauge fixing. From the definition of the observables given above, a standard argument reveals that they correspond to cohomology classes on the moduli space, denoted by $\mathcal{M}$. In particular, a BRST invariant observable $W^{(i, 4-i)}$ reduces to a closed form on $\mathcal{M}$ of degree $(4-i)$. The vacuum expectation of a product of observables then has an interpretation as an intersection number on $\mathcal{M}$, for details see [32, 33].

The space of interest here is given by the flat connections, $R_{\mu \nu}=0$. Of course, compact manifolds which admit flat connections in the euclidean case are rather rare, and we will later modify this choice by introducing curvature singularities. The other condition that we need to impose at this point is perhaps more subtle [4]. If we consider the transformation of the spin connection under the combined shift and local Lorentz symmetries (see (14)),

$$
\delta \omega_{\mu}^{r s}=\epsilon R_{\theta \mu}^{r s}+D_{\mu} \epsilon^{r s},
$$

we see that some deformations in the vielbein lead to trivial deformations in the connection, in the sense that they can be absorbed into a Lorentz gauge transformation. If we impose the constraint

$$
\hat{D}^{\mu} R_{\theta \mu}=0
$$

on all such deformations, then - in the absence of zero modes of the Laplacian when acting on scalars in the adjoint representation - we eliminate these trivial deformations. Since there is a one-to-one correspondence between scalars in the adjoint representation and 2-forms, namely $\phi_{\mu \nu} \equiv h_{\mu}^{i} h_{\nu}^{j} \phi_{i j}$, the Hodge theorem shows that the number of such zero modes is equal to the dimension of the second cohomology group $H^{2}(M, \mathbf{R})$. Here, $\hat{D}_{\mu}$ is the full covariant derivative defined with respect to the spin and Christoffel connections by

$$
\hat{D}_{\mu} X_{\nu}^{i}=\partial_{\mu} X_{\nu}^{i}-\omega_{\mu j}^{i} X_{\nu}^{j}-\Gamma_{\mu \nu}^{\rho} X_{\rho}^{i},
$$

and the curvature components are given by

$$
R_{\mu \nu}{ }^{r s}=h_{\mu}^{i} h_{\nu}^{j} R_{i j}{ }^{r s}, R_{\theta \mu}{ }^{r s}=h_{\mu}^{i} R_{\theta i}{ }^{r s} .
$$

Although we are not dealing with a theory of an independent spin connection, we are, nevertheless, interested in some moduli space of Riemannian connections. While the above conditions are clearly motivated by the construction of topological gauge theory, there are crucial differences here which simply reflect the fact that in Riemannian geometry one constructs the connection from the vielbein (or equivalently, the metric).

To illuminate the structure of this action, we require a little more armoury. It is straightforward to derive the following BRST-SUSY transformations, see (33) and (34):

$$
\begin{aligned}
\delta R_{\theta \mu}{ }^{r s} & =\frac{1}{2} \epsilon \hat{D}_{\mu} \phi^{r s}-\epsilon^{a} \delta_{a}^{i} h_{i}^{\nu} R_{\mu \nu}{ }^{r s}, \\
\delta R_{\mu \nu}{ }^{r s} & =\epsilon \hat{D}_{[\mu} R_{\theta \nu]}{ }^{r s} .
\end{aligned}
$$

In addition, we have

$$
\delta h_{i}^{\mu}=-\epsilon h_{i}^{\nu} \psi_{\nu}^{a} \delta_{a}^{j} h_{j}^{\mu}, \delta \sqrt{g}=\epsilon \sqrt{g} h_{i}^{\mu} \psi_{\mu}^{a} \delta_{a}^{i}, \delta \Gamma_{\mu \nu}^{\mu}=\epsilon h_{i}^{\mu} \delta_{a}^{i} \hat{D}_{\nu} \psi_{\mu}^{a},
$$


where the final transformation is obtained by studying the condition for covariant constancy of the vielbein, namely: $\hat{D}_{\mu} h_{\nu}^{i}=0$. We record the BRST variation of the constraint $\hat{D}^{\mu} R_{\theta \mu}$ for future reference,

$$
\begin{aligned}
Q_{B R S T}\left(\hat{D}_{\mu} R_{\theta}{ }^{\mu r s}\right) & =\frac{1}{2} \hat{D}^{2} \phi^{r s}-R_{\theta \mu}{ }^{l m} R_{\theta}^{\mu p q} f_{[l m][p q]}^{[r s]}-R_{\theta}{ }^{\mu r s} h_{i}^{\nu} \delta_{a}^{i} T_{\mu \nu}^{a} \\
& +R_{\theta i}{ }^{r s} \delta_{a}^{i} \hat{D}^{\mu} \psi_{\mu}^{a}+h^{\mu i} \delta_{i a} g^{\nu \lambda} \psi_{\lambda}^{a} \hat{D}_{(\mu} R_{\nu) \theta}{ }^{r s}
\end{aligned}
$$

Let us now concentrate our attention on a specific model. In two dimensions, the vector representation of $\mathrm{SO}(2)$ is reducible, so we have automatically $N=2$ (the number of gravitinos) vector supergravity. In the present instance, we choose $V$ to be:

$$
V=\epsilon^{\mu \nu} \chi R_{\mu \nu}+\sqrt{g} \bar{\phi} \hat{D}_{\mu} R_{\theta}^{\mu}
$$

and therefore we have manifest invariance with respect to Lorentz rotations, and diffeomorphisms. The symmetries of the minimal multiplet have already been given, and we need only specify the transformations of the antighosts $(\chi, \bar{\phi})$, and their multipliers, which we denote by $(B, \eta)$. These transformations must be such that they obey the closure properties of the BRST-SUSY algebra, and guarantee that the action is invariant under vector supersymmetry. This can be achieved, and one set is as follows:

$$
\begin{aligned}
\delta \bar{\phi} & =\epsilon \eta \\
\delta \eta & =\epsilon^{a} \delta_{a}^{i} D_{i} \bar{\phi} \\
\delta \chi & =\epsilon B+\frac{1}{2} \epsilon^{a} \delta_{a}^{i} \epsilon_{i}^{j} D_{j} \bar{\phi}, \\
\delta B & =\epsilon^{a} \delta_{a}^{i}\left(D_{i} \chi-\frac{1}{2} \epsilon_{i}{ }^{j} D_{j} \eta\right) .
\end{aligned}
$$

For convenience, we have presented the above transformations in flat indices; we note that the flat and curved epsilon symbols are generally related by

$$
\operatorname{det}\left[h_{\mu}^{i}\right] \epsilon^{i_{1} i_{2} \cdots}=\epsilon^{\mu_{1} \mu_{2} \cdots} h_{\mu_{1}}^{i_{1}} h_{\mu_{2}}^{i_{2}} \cdots .
$$

The SUSY transformations of $\chi$ and $\bar{\phi}$ were determined by requiring $V$ to transform as a total derivative under that symmetry, and the others are then fixed by closure of the original algebra.

Expanding (56), we obtain

$$
\begin{aligned}
S_{q} & =\int\left\{\epsilon^{\mu \nu}\left(B R_{\mu \nu}-\chi \hat{D}_{[\mu} R_{\theta \nu]}\right)+\sqrt{g}\left(\eta+h_{i}^{\nu} \psi_{\nu}^{a} \delta_{a}^{i} \bar{\phi}\right) \hat{D}_{\mu} R_{\theta}^{\mu}+\sqrt{g} \bar{\phi}\left(\frac{1}{2} \hat{D}^{2} \phi\right.\right. \\
& \left.\left.-R_{\theta}{ }^{\mu} h_{i}^{\nu} \delta_{a}^{i} T_{\mu \nu}^{a}+R_{\theta i} \delta_{a}^{i} \hat{D}^{\mu} \psi_{\mu}^{a}+h^{\mu i} \delta_{i a} g^{\nu \lambda} \psi_{\lambda}^{a} \hat{D}_{(\mu} R_{\nu) \theta}\right)\right\} .
\end{aligned}
$$

The first point to note here is that the expansion of the topological gauge fixing condition generates the second term in (67). One then notices that $R_{\theta \mu}$ itself behaves as a gauge field, and thus requires gauge fixing. This is effected by the second gauge fixing in (64).

In order to proceed in dimensions greater that two, one simply replaces $\chi$ and $B$ by $(n-2)$-forms. However, for certain purposes it is convenient to introduce the associated tensor densities $\bar{B}^{\mu \nu}$ and $\bar{\chi}^{\mu \nu}$, defined as follows:

$$
\begin{aligned}
& \bar{B}^{\mu_{1} \mu_{2}}=\epsilon^{\mu_{1} \mu_{2} \mu_{3} \cdots \mu_{n}} B_{\mu_{3} \cdots \mu_{n}}, \\
& \bar{\chi}^{\mu_{1} \mu_{2}}=\epsilon^{\mu_{1} \mu_{2} \mu_{3} \cdots \mu_{n}} \chi_{\mu_{3} \cdots \mu_{n}} .
\end{aligned}
$$


The form of the quantum action which is generic to all dimensions is given by the action of $Q_{B R S T}$ on

$$
V=\operatorname{tr}\left(\bar{\chi}^{\mu \nu} R_{\mu \nu}+\sqrt{g} \bar{\phi} \hat{D}_{\mu} R_{\theta}^{\mu}\right)
$$

and again we have manifest invariance with respect to both Lorentz rotations, and diffeomorphisms. However, invariance under vector supersymmetry requires a little extra attention, but can be achieved with the following set of transformations:

$$
\begin{aligned}
\delta \bar{\phi} & =\epsilon \eta, \\
\delta \eta & =-\frac{1}{2} \epsilon[\phi, \bar{\phi}]+\epsilon^{a} \delta_{a}^{i} h_{i}^{\mu} \hat{D}_{\mu} \bar{\phi}, \\
\delta \bar{\chi}^{\mu \nu} & =\epsilon \bar{B}^{\mu \nu}+\frac{1}{2} \sqrt{g} \epsilon^{a} \delta_{a}^{i} h_{i}^{[\mu} \hat{D}^{\nu]} \bar{\phi}, \\
\delta \bar{B}^{\mu \nu} & =-\frac{1}{2} \epsilon\left[\phi, \bar{\chi}^{\mu \nu}\right]-\epsilon^{a} \delta_{a}^{i} h_{i}^{[\mu}\left\{\hat{D}_{\lambda} \bar{\chi}^{\nu] \lambda}+\frac{1}{2} \sqrt{g} Y^{\nu]}\right\},
\end{aligned}
$$

where

$$
Y_{\rho}=\hat{D}_{\rho} \eta+\left[R_{\theta \rho}, \bar{\phi}\right]-h_{j}^{\sigma} \psi_{\rho}^{b} \delta_{b}^{j} \hat{D}_{\sigma} \bar{\phi}+h_{j}^{\sigma} \psi_{\sigma}^{b} \delta_{b}^{j} \hat{D}_{\rho} \bar{\phi}-h_{\rho j} \psi_{\sigma}^{b} \delta_{b}^{j} \hat{D}^{\sigma} \bar{\phi}
$$

For the case of three dimensions, we find

$$
\begin{aligned}
S_{q} & =\int \operatorname{tr}\left\{\epsilon^{\rho \mu \nu}\left(B_{\rho} R_{\mu \nu}-\chi_{\rho} \hat{D}_{[\mu} R_{\theta \nu]}\right)+\sqrt{g}\left(\eta+h_{i}^{\nu} \psi_{\nu}^{a} \delta_{a}^{i} \bar{\phi}\right) \hat{D}_{\mu} R_{\theta}{ }^{\mu}+\sqrt{g} \bar{\phi}\left(\frac{1}{2} \hat{D}^{2} \phi\right.\right. \\
& \left.\left.+\left[R_{\theta \mu}, R_{\theta}{ }^{\mu}\right]-R_{\theta}{ }^{\mu} h_{i}^{\nu} \delta_{a}^{i} T_{\mu \nu}^{a}+R_{\theta i} \delta_{a}^{i} \hat{D}^{\mu} \psi_{\mu}^{a}+h_{i}^{\mu} \delta_{a}^{i} g^{\nu \lambda} \psi_{\lambda}^{a} \hat{D}_{(\mu} R_{\nu) \theta}\right)\right\}
\end{aligned}
$$

where we note the presence of the extra commutator term in $R_{\theta \mu}$.

One feature that arises here (and in all dimensions greater than two) is that the $\chi$ and $B$ fields possess their own local symmetries; in the case at hand, one finds that $S_{q}$ is invariant under

$$
\delta \chi_{\mu}=D_{\mu} \Lambda^{(1)}, \delta B_{\mu}=D_{\mu} \Lambda^{(0)}+\left[R_{\theta \mu}, \Lambda^{(1)}\right],
$$

where $\Lambda^{(0)}$ and $\Lambda^{(1)}$ are even and odd transformation parameters. The closure of the algebra on the $\chi_{\mu}$ and $B_{\mu}$ fields can be performed, and one again verifies the off-shell nature of this algebra. We simply note that the $\left[\delta_{S U S Y}, \delta_{B R S T}\right]$ commutator defines a diffeomorphism of these fields, and the remaining algebra proceeds obediently.

We can now make direct contact with the universal bundle for gravity. Independent of the particular dimension, we see that the $\bar{\phi}$ equation of motion asserts that the $\phi$ component of the curvature assumes its expected non-local form:

$$
\begin{aligned}
\phi^{r s}= & -2\left(\hat{D}^{2}\right)^{-1}\left(-R_{\theta \mu}{ }^{l m} R_{\theta}^{\mu p q} f_{[l m][p q]}^{[r s]}-R_{\theta}{ }^{\mu r s} h_{i}^{\nu} \delta_{a}^{i} T_{\mu \nu}^{a}\right. \\
& \left.+R_{\theta i}{ }^{r s} \delta_{a}^{i} \hat{D}^{\mu} \psi_{\mu}^{a}+h^{\mu i} \delta_{i a} g^{\nu \lambda} \psi_{\lambda}^{a} \hat{D}_{(\mu} R_{\nu) \theta}{ }^{r s}\right) .
\end{aligned}
$$

Furthermore, the middle component $R_{\theta \mu}$ also obeys the necessary horizontality condition, $\hat{D}_{\mu} R_{\theta}^{\mu}=0$. This shows that the above cohomological gravity theory provides a field theoretic representation [4] of a universal bundle for gravity in any dimension [17, 29].

It was observed in [28] that as a consequence of the form of the quantum action in (56), the theory naturally contains a vector supersymmetry operator. The energy-momentum tensor obtained from $S_{q}$ has the form

$$
T_{\mu \nu}=\left\{Q_{B R S T}, V_{\mu \nu}\right\} .
$$

Defining the momentum operator $P_{\mu}$, and its BRST partner $V_{\mu}$, by integrating $T_{0 \mu}$ and $V_{0 \mu}$ over appropriate spacelike hypersurfaces, one obtains the algebra:

$$
P_{\mu}=\left\{Q_{B R S T}, V_{\mu}\right\}
$$


Clearly, we can identify $V_{\mu}$ as the vector supersymmetry operator, and the above argument indicates that cohomological theories will always contain such a symmetry. It was also shown in [28], that the descent equations follow as a result of the defining relation (76).

The approach we have adopted here is to define cohomological theories at the algebraic level, independent of any particular model. Indeed, as we have seen, one can obtain a realization of the BRST-SUSY algebra and define an observable hierarchy at this level, prior to the specification of any moduli space.

\section{Uniformization Approach to Cohomological Grav- ity}

In performing the topological gauge fixing, we restricted ourselves to the condition of flat curvature, $R_{\mu \nu}=0$. We were able to impose this condition through the introduction of a multiplier field $\mathrm{B}$, and the resulting action was observed to have local supersymmetry. This same differential form B does, however, allow us to introduce curvature singularities on codimension two submanifolds. This path was also adopted by Verlinde and Verlinde [22] in two dimensions. In this way, vector supersymmetry, as well as diffeomorphisms, are broken only along these punctures. It is important to stress that the algebra (36) is defined at the equivariant level, in which the local symmetries of Lorentz, diffeomorphisms, and vector supersymmetry are still manifest. Indeed, we shall adopt the equivariant approach as a guiding principle for defining the theory. As in any gauge theory, one should be able to define the action, observables, and path integral at this level, prior to the gauge fixing of the local symmetries.

For the case of three dimensions, the puncture operator corresponds to a Wilson loop defined by

$$
W_{B}(C)=\operatorname{tr} P \exp \oint_{C} B
$$

where $P$ denotes the path ordering, and $C$ is a closed 1 -cycle. This operator is a solution to the differential equation

$$
\left(\frac{d}{d t}+M(t)\right) W_{B}(t)=0
$$

where

$$
M(t)=B_{\mu}(x(t)) \dot{x}^{\mu}(t) .
$$

To obtain the Wilson loop in (77), we have chosen a closed path $C$, with $x(0)=x(2 \pi)$, and taken a Lorentz trace. Inserting a product of these operators in the path integral measure leads to a modified equation of motion for the $B$ field. We find that the resulting moduli space is given by flat Riemannian connections, with delta function singularities defined along the codimension two punctures. One can thus view the above analysis as a uniformization approach to different moduli spaces. The vacuum expectation values of the observables presented in section 5 will then correspond to cohomology classes on these moduli spaces. In dimension greater than three, one notes that the puncture operators become Wilson "surfaces".

Since we are working at the equivariant level, we must ensure the BRST, and Lorentz invariance of the functional measure. The Wilson loop is clearly invariant with respect to the latter symmetry, while we see from (70) that it is only BRST invariant if we restrict the $\phi$ field to vanish along the loop $C$. Specifically, we have the following formula for a 
general variation of the puncture operator [34]:

$$
\delta \operatorname{tr} P \exp \int_{0}^{2 \pi} M(\tau) d \tau=\int_{0}^{2 \pi} d t \operatorname{tr}\{\delta M(t) W[2 \pi, t] W[t, 0]\}
$$

where

$$
W[b, a]=P \exp \int_{a}^{b} M(t) d t .
$$

From (70), the BRST variation is given by

$$
Q_{B R S T} M(t)=-\frac{1}{2}\left[\phi, \chi_{\mu}\right] \dot{x}^{\mu}
$$

and the simplest option, and the only one we will consider here, is to demand that $\phi$ vanish on $C$, in order to achieve BRST invariance of the puncture insertion. Moreover, the very presence of the puncture insertions dictates the relevant diffeomorphism group of the theory to be one which leaves fixed these codimension two submanifolds. As a result, the local vector supersymmetry is similarly restricted, and one notes that the local $B$, and hence $\chi$, symmetries are also broken on the punctures.

The correlation functions of the theory are defined by:

$$
Z\left(\gamma_{1}, \ldots, \gamma_{r} ; C_{1}, \ldots, C_{k}\right)=\int[d \Phi] \prod_{i=1}^{k} W_{B}\left(C_{i}\right) e^{i S_{q}} \prod_{j=1}^{r} W\left(\gamma_{j}\right)
$$

where $\Phi$ denotes the collective field content. In order to obtain a non-vanishing result, one must ensure that the ghost number of the correlator equals the dimension of the moduli space under discussion (or equivalently, the ghost number anomaly). The generic situation in cohomological models is one in which there are ghost zero modes; this may result in a net ghost number violation in the path integral measure, and hence to a ghost number anomaly. As we have seen, the observables have positive ghost number, and hence in the presence of the anomaly one inserts a collection of observables whose total ghost number equals the anomaly. At the level of differential forms on moduli space $\mathcal{M}$, this translates into the condition that the total ghost number of the observable be equated with the dimension of $\mathcal{M}$.

With this restriction on the $\phi$ field, we should re-examine the 0 - and 1 -form observables given above. It appears that the first observable becomes trivial in the presence of puncture insertions; this follows from the fact that as a result of the descent equation (41), the observable is independent of its position, and hence one can always move it to a point on the puncture where $\phi$ vanishes. This simple argument assumes, of course, that there are no subtleties involved in placing $\phi$ on the punctures. However, the case for the 1form $W^{(1,3)}$ requires closer attention. In this regard, we note that the variation of $W(C)$ with respect to an infinitesimal variation of the loop is equal to a BRST commutator. Explicitly, we have

$$
\delta M(t)=\delta x^{\nu}\left(\partial_{\nu} B_{\mu}\right) \dot{x}^{\mu}+B_{\mu} \frac{d}{d t} \delta x^{\mu}
$$

and hence

$$
\begin{aligned}
\delta W_{B}(C) & =\int_{0}^{2 \pi} d t \operatorname{tr}\left\{\left(\partial_{[\mu} B_{\nu]}+\left[B_{\mu}, B_{\nu}\right]\right) \delta x^{\mu} \dot{x}^{\nu} W[2 \pi, t] W[t, 0]\right\} \\
& =Q_{B R S T}\left(\int_{0}^{2 \pi} \operatorname{tr}\left\{D_{B[\mu} \chi_{\nu]} \delta x^{\mu} \dot{x}^{\nu} W[2 \pi, t] W[t, 0]\right\}\right)
\end{aligned}
$$


where the latter step is achieved by restricting $\phi$ to vanish on $C$. In the above, we have defined a 'covariant' derivative with respect to $B$, namely $D_{B \mu} \chi_{\nu}=\partial_{\mu} \chi_{\nu}+\left[B_{\mu}, \chi_{\nu}\right]$. This establishes the fact that the puncture insertions are invariant under small deformations of the loop. Nevertheless, it is possible to examine the 1 -form observable for a homology cycle which is not homologous to the puncture loop, and such an observable may well be non-trivial. The issue of whether there is a ghost number anomaly in three dimensions - which is essential if one is to compute nonzero values for observables other than the partition function - will be considered in the next section along with the gauge theory construction.

It is worth making a few remarks on the situation in two dimensions. Since the rotation group in this case is Abelian, the BRST operator acting on a field in the adjoint representation is nilpotent at the equivariant level. Consequently, the $B$ field is BRST invariant, (see (65)), and one does not encounter a restriction on the $\phi$ field; in addition, the puncture operators are independent of their positions. Indeed, it has been shown that the 0 -form operators do, in fact, correspond to cohomology classes on the moduli space of punctured Riemann surfaces [32, 33], [22].

Of course, in order to fully define the path integral, one must proceed with the gauge fixing of all the local symmetries. This leads to a total BRST operator which is obtained by replacing all the local parameters by the old BRST parameter $\epsilon$, times the corresponding ghost field. One then introduces suitable gauge fixing conditions to break diffeomorphisms, frame rotations, and local vector supersymmetry. In dimension greater than two, one must also break the $B$ and $\chi$ local invariances. In this way, the action (67) or (72) is bedecked with the full tapestry of ghost and multiplier fields. Suffice it to say at this point that the entire field content is such that to each Grassmann even field, one has a Grassmann odd partner of identical field type. In addition to the fields $\left(h_{\mu}^{i}, \psi_{\mu}^{a}\right)$, and $\left(\phi^{r s}, \bar{\phi}^{r s}, \eta^{r s}\right)$, one generically requires three sets of (ghost, antighost, multiplier) fields. These are given by $\left(c^{i}, \bar{c}^{i}, b^{i}\right),\left(c^{a}, \bar{c}^{a}, b^{a}\right),\left(c^{r s}, \bar{c}^{r s}, b^{r s}\right)$. Respectively, these are present for the diffeomorphism, vector supersymmetry, and local Lorentz invariances. Thus, one sees that the diffeomorphism ghosts cancel against the supersymmetry ghosts, while the Lorentz ghosts play host to the second stage ghosts $(\phi, \bar{\phi})$. Additionally, the ghosts for the $B$ and $\chi$ symmetry form a supersymmetric set. It is clear that by gauge fixing all the local symmetries, one will obtain a total BRST operator, $Q_{T O T A L}$, which is nilpotent. One then has the freedom to choose the Lagrangian to be exact with respect to either the original $Q_{B R S T}$ as in (56), or the complete $Q_{T O T A L}$.

We present here the $Q_{\text {TOTAL }}$ transformations of the fundamental multiplet; this can be read off from (33) and (14):

$$
\begin{aligned}
\delta h_{\mu}^{i} & =\epsilon\left(D_{\mu} c^{i}+\delta_{a}^{i} \psi_{\mu}^{a}+c^{i j} \delta_{j k} h_{\mu}^{k}\right), \\
\delta \psi_{\mu}^{a} & =\epsilon\left(D_{\mu} c^{a}-h_{\mu}^{i} T_{i \theta}^{a}+c^{i} T_{i j}^{a} h_{\mu}^{j}+c^{i j} \delta_{i}^{a} \delta_{b j} \psi_{\mu}^{b}\right), \\
\delta \phi^{r s} & =\epsilon\left(2 c^{a} \delta_{a}^{i} R_{\theta i}{ }^{r s}+c^{i} D_{i} \phi^{r s}-[c, \phi]^{r s}\right) .
\end{aligned}
$$

Nilpotency then dictates the following ghost transformations:

$$
\begin{aligned}
\delta c^{i} & =\epsilon\left(-\delta_{a}^{i} c^{a}+c^{i j} c_{j}\right), \\
\delta c^{i j} & =\epsilon\left(-\frac{1}{2}(\phi+[c, c])^{i j}-c^{k} R_{\theta k}{ }^{i j}+\frac{1}{2} c^{l} c^{m} R_{l m}{ }^{i j}\right), \\
\delta c^{a} & =\epsilon\left(-\frac{1}{2} \delta_{i}^{a} \phi^{i j} c_{j}+\delta_{i}^{a} c^{k} c_{j} R_{\theta k}{ }^{i j}+\delta_{i}^{a} c^{i j} \delta_{j b} c^{b}\right) .
\end{aligned}
$$

The condition that the symmetries are broken on the codimension two punctures is encoded in the path integral by restricting the relevant ghost fields to vanish along these 
punctures. It is worth remarking that once the full BRST operator is obtained, a new BRST invariant $B$ field can be constructed. This is achieved by shifting the old $B$ field by certain ghost terms. Therefore, having fully gauge fixed the theory, one no longer sees the restriction on the $\phi$ field, nor on the diffeomorphism group. As mentioned previously, a necessary consequence of the equivariant point of view is that in order to implement curvature singularities, one is forced to restrict the $\phi$ field on the punctures.

\section{Uniformization Approach to Gauge Theory}

Having discussed the uniformization approach to defining cohomological gravity, it is useful to return to the gauge theory situation. In particular, let us examine a gauge theory in three dimensions; this model is known as a super- $B F$ system [35, 21], and it has been established that the partition function yields the Casson invariant [35]. Let us first describe the general features of this system; the BRST-SUSY transformations are as follows [1]:

$$
\begin{aligned}
\delta A_{\mu} & =\epsilon \psi_{\mu}, \delta \psi_{\mu}=\frac{\epsilon}{2} D_{\mu} \phi+\epsilon^{\nu} F_{\nu \mu}, \delta \phi=2 \epsilon^{\mu} \psi_{\mu}, \\
\delta \bar{\phi} & =\epsilon \eta, \delta \eta=-\frac{1}{2} \epsilon[\phi, \bar{\phi}]+\epsilon^{\mu} D_{\mu} \bar{\phi}, \delta \chi_{\mu}=\epsilon B_{\mu}+\frac{1}{2} \epsilon^{\nu} \epsilon_{\mu \nu}{ }^{\rho} D_{\rho} \bar{\phi} \\
\delta B_{\mu} & =-\frac{1}{2} \epsilon\left[\phi, \chi_{\mu}\right]-\epsilon^{\nu}\left\{D_{[\mu} \chi_{\nu]}+\frac{1}{2} \epsilon_{\mu \nu}{ }^{\rho}\left(D_{\rho} \eta+\left[\psi_{\rho}, \bar{\phi}\right]\right)\right\}
\end{aligned}
$$

where $\psi_{\mu}=\delta_{\mu}^{i} \psi_{i}$, and $\epsilon^{\mu}=\delta_{i}^{\mu} \delta_{a}^{i} \epsilon^{a}$. It is straightforward to check the closure of the associated algebra [1].

The quantum action for this theory can be written as $[35,21]$

$$
S_{q}=\int Q_{B R S T} \operatorname{tr}\left(\epsilon^{\rho \mu \nu} \chi_{\rho} F_{\mu \nu}+\bar{\phi} D_{\mu} \psi^{\mu}\right)
$$

which, upon expansion, becomes

$$
S_{q}=\int \operatorname{tr}\left\{\epsilon^{\rho \mu \nu}\left(B_{\rho} F_{\mu \nu}-\chi_{\rho} D_{[\mu} \psi_{\nu]}\right)+\eta D_{\mu} \psi^{\mu}+\bar{\phi}\left(\frac{1}{2} D^{2} \phi+\left[\psi_{\mu}, \psi^{\mu}\right]\right)\right\} .
$$

It is easy to verify invariance with respect to global vector supersymmetry transformations, while the BRST symmetry is manifest. In addition, one finds invariance with respect to the following $B$ and $\chi$ gauge transformations:

$$
\delta \chi_{\mu}=D_{\mu} \Lambda^{(1)}, \delta B_{\mu}=D_{\mu} \Lambda^{(0)}+\left[\psi_{\mu}, \Lambda^{(1)}\right]
$$

where $\Lambda^{(0)}$ and $\Lambda^{(1)}$ are gauge parameters in the adjoint representation of $G$. It is worth noting the BRSY-SUSY algebra closes on $\chi$ and $B$ in the following manner:

$$
\begin{aligned}
& {\left[\delta_{S U S Y}\left(\epsilon^{\nu}\right), \delta_{B R S T}(\epsilon)\right] \chi_{\mu}=\epsilon \epsilon^{\nu} D_{\nu} \chi_{\mu}+D_{\mu}\left(-\epsilon \epsilon^{\nu} \chi_{\nu}\right)} \\
& {\left[\delta_{S U S Y}\left(\epsilon^{\nu}\right), \delta_{B R S T}(\epsilon)\right] B_{\mu}=\epsilon \epsilon^{\nu} D_{\nu} B_{\mu}+D_{\mu}\left(-\epsilon \epsilon^{\nu} B_{\nu}\right)+\left[\psi_{\mu},-\epsilon \epsilon^{\nu} \chi_{\nu}\right] .}
\end{aligned}
$$

In other words, the SUSY-BRST commutator closes up to a translation and a local $B-\chi$ gauge transformation.

The above field theory, like any other cohomological gauge theory based upon this multiplet, provides a field theoretic representation of the universal bundle of Atiyah and 
Singer [17]. Specifically, one notes from the $\bar{\phi}$ equation of motion that the non-local structure of the $\phi$ component of the universal curvature is dictated to be:

$$
\phi=-2\left(D^{2}\right)^{-1}\left[\psi_{\mu}, \psi^{\mu}\right] .
$$

In addition, the $\eta$ equation of motion ensures that $\psi$ obeys the required horizontality condition $D \cdot \psi=0$. These are exactly the formulae obtained in the analysis of Atiyah and Singer.

In order to consider the observables in this theory, we note that the net ghost number violation is zero. It appears therefore that any observable, beyond the partition function, vanishes. This feature arises precisely in three dimensions because of the fact that the antighost $\chi_{\mu}$ is a 1 -form of ghost number -1 , and satisfies the same equation as $\psi_{\mu}$. Therefore, the number of $\psi$ zero modes will equal those of $\chi$. Furthermore, the multiplier fields enforcing the gauge fixing of both $\psi$ and $\chi$ will also have identical zero modes, and one finds that the ghost number anomaly is absent.

One can also adopt the uniformization approach to topological gauge theory. In three dimensions, the theory naturally provides a 1 -form field $B_{\mu}$ in the adjoint representation of $G$, and we can then consider the associated Wilson loop (77), with a $G$ trace replacing the Lorentz trace. As before, the presence of this puncture operator modifies the moduli space condition to be one of flat gauge connections, with curvature singularities defined along the codimension two punctures. We also see that the BRST invariance of the puncture operator requires the vanishing of $\phi^{I}$ along the loop $C$. As long as $G$ is non-Abelian, this will be the case in any dimension; additionally, the $B$ and $\chi$ local symmetries will also be broken on the punctures.

Unfortunately, it appears that the presence of these punctures does not overcome the absence of a ghost number anomaly. Since $\phi$ vanishes on the punctures, and the $\chi$ symmetry is also broken there, one sees that the gauge fixing for both the $\psi$ and $\chi$ symmetry is identical, and hence their respective multiplier fields will be restricted in the same way. Nevertheless, the partition function for the case of flat connections with singularities defined on codimension two punctures is certainly worthy of attention.

Let us note that one can complete the identification with the universal bundle by performing the gauge fixing of the Yang-Mills symmetry. The transformations of the minimal multiplet now take the form

$$
\begin{aligned}
\delta A_{\mu} & =\epsilon\left(\psi_{\mu}+D_{\mu} c\right), \\
\delta \psi_{\mu} & =\epsilon\left(\frac{1}{2} D_{\mu} \phi-\left[c, \psi_{\mu}\right]\right)+\epsilon^{\nu} F_{\nu \mu}, \\
\delta \phi & =-\epsilon[c, \phi]+2 \epsilon^{\mu} \psi_{\mu},
\end{aligned}
$$

where $c$ is the Yang-Mills ghost; nilpotency of the resulting BRST operator specifies the following transformation for this ghost:

$$
\delta c=-\frac{1}{2} \epsilon(\phi+[c, c])+\epsilon^{\mu} A_{\mu} .
$$

The algebra is now given by:

$$
\left[\delta_{S U S Y}\left(\epsilon^{\mu}\right), \delta_{B R S T}(\epsilon)\right]=\epsilon \epsilon^{\mu} \partial_{\mu}
$$

Once we have introduced the ghost field, we must specify its variation under global vector supersymmetry, and in order to achieve closure of the algebra, this dictates the above 
transformation. The interesting point here is to note that the vector supersymmetry operator behaves as a shift operator connecting the two components of the connection of the universal bundle, see (28):

$$
\delta_{S U S Y}: c \rightarrow A \rightarrow 0
$$

Returning to the issue of the ghost number anomaly, we find that the situation in gravity is somewhat more involved, since $R_{\theta \mu}$ is not an independent field in the theory. As we have seen, it represents a deformation in the spin connection which is consistent with the no torsion constraint. In addition, the gravitino $\psi_{\mu}^{a}$ is the gauge field for local supersymmetry, and this has no counterpart in the simple gauge theory setting. Even in three dimensions, where $\chi_{\mu}^{r s}$ and $\psi_{\mu}^{r s}=\epsilon^{r s i} \delta_{i a} \psi_{\mu}^{a}$ have the same index structure, these two fields are of different geometrical type. The ghost number anomaly in this formulation of cohomological gravity requires a more careful treatment, and we will not undertake that here.

\section{Outlook}

We have presented an algebraic foundation for both cohomological gauge and gravity theories. The gauge theories are based on a certain global vector supersymmetry, while local supersymmetry turns out to be the essential ingredient in the gravity models. Our construction is, moreover, generic to all dimensions. Adopting a uniformization approach, where curvature singularities are implemented on codimension two punctures, is natural within this framework.

In the $3 \mathrm{~d}$ gravity model, it would be interesting to develop the theory on manifolds with boundary. The boundary components are Riemann surfaces, and one knows that here there are observables which correspond to the Mumford invariants. The role of the three dimensional theory in interpolating between the boundary components needs to be elucidated. For $3 \mathrm{~d}$ gauge theory, the partition function in the presence of puncture insertions has not been studied. Whether it represents an interesting extension of the Casson invariant is an open issue.

It is known that one can triangulate any manifold in such a way that the Riemann curvature is everywhere vanishing, except for singularities defined on submanifolds of codimension two. With this knowledge, a discrete Regge version of the theory might be constructed. The fact that a form $B$, of co-degree two, is naturally provided in the continuum cohomological theory, suggests that it may well be possible to actually compute the observables of the theory through a discrete Regge analysis. An important observation in this regard is the fact that all the fields in the cohomological model are differential forms, either even or odd, of varying degree. Moreover, one sees that a "fermion" doubling problem will not arise for the Grassmann odd forms [36]. One might envisage a thorough Regge analysis of the two dimensional model without recourse to conformal field theory, or topological methods [22], [32, 33]. Our prime concern is, however, the study of cohomological gravity in higher dimensions, and the formulation we have presented here provides a foundation for pursuing this objective. 


\section{References}

[1] D. Birmingham and M. Rakowski, Phys. Lett. 272B (1991) 217.

[2] D. Birmingham and M. Rakowski, Phys. Lett. 272B (1991) 223.

[3] D. Birmingham and M. Rakowski, Phys. Lett. 273B (1991) 74.

[4] D. Birmingham and M. Rakowski, Phys. Lett. 275B (1992) 289.

[5] E. Witten, Commun. Math. Phys. 117 (1988) 353.

[6] E. Witten, Phys. Lett. 206B (1988) 601.

[7] J.M. Labastida, M. Pernici and E. Witten, Nucl. Phys. B310 (1988) 611.

[8] D. Montano and J. Sonnenschein, Nucl. Phys. B324 (1989) 348.

[9] R. Myers and V. Periwal, Nucl. Phys. B333 (1990) 536.

[10] R. Myers, Nucl. Phys. B343 (1990) 705; Phys. Lett. 252B (1990) 365.

[11] C.G. Torre, Phys. Lett. 252B (1990) 242.

[12] H. Kunitomo, Mod. Phys. Lett. A6 (1990) 2389.

[13] A. Nakamichi, I. Oda and A. Sugamoto, Phys. Rev. D44 (1991) 3835.

[14] K. Ito, Topological field theory of four dimensional self-dual gravity and twisted harmonic superspace, Univ. Tokyo preprint, August 1991.

[15] L. Baulieu and I.M. Singer, Commun. Math. Phys. 135 (1991) 253.

[16] S. Wu, Four dimensional topological gravity and the universal fibration of Riemannian manifolds, MSRI-04929-91, June 1991.

[17] M.F. Atiyah and I.M. Singer, Proc. Nat'l. Acad. Sci. (USA) 81 (1984) 2597.

[18] L. Baulieu and I.M. Singer, Nucl. Phys. B (Proc. Suppl.) 5B (1988) 12.

[19] D. Birmingham, M. Rakowski and G. Thompson, Nucl. Phys. B315 (1989) 577.

[20] H. Kanno, Z. Phys. C43 (1989) 477.

[21] D. Birmingham, M. Blau and G. Thompson, Int. J. Mod. Phys. A5 (1990) 4721.

[22] E. Verlinde and H. Verlinde, Nucl. Phys. B348 (1991) 457.

[23] S. MacDowell, Phys. Lett. 80B (1979) 212.

[24] T. Regge, Nuovo Cim. 19 (1961) 558.

[25] S. MacDowell, Phys. Lett. 80 B (1979) 212; S. MacDowell, in Supergravity, eds. P. van Nieuwenhuizen and D. Freedman (North Holland) 1979; S. MacDowell, in VI Brazilian Symposium on Theoretical Physics, Vol. III, eds. Ferreira and B. Koiller (CNPq, Coordenacao Editorial Brasilia) 1981. 
[26] S. MacDowell and M. Rakowski, Nucl. Phys. B274 (1986) 589; Errata in Nucl. Phys. B282 (1987) 747.

[27] S.J. Gates Jr., M.T. Grisaru, M. Roček and W. Siegel, Superspace, Benjamin/Cummings, Reading, Mass. 1983.

[28] R. Dijkgraaf, E. Verlinde and H. Verlinde, Nucl. Phys. B352 (1991) 59.

[29] O. Alvarez, I.M. Singer and B. Zumino, Commun. Math. Phys. 96 (1984) 409.

[30] J.M.F. Labastida and M. Pernici, Phys. Lett. 213B (1988) 319.

[31] R. Brooks, D. Montano and J. Sonnenschein, Phys. Lett. 214B (1988) 91.

[32] E. Witten, Nucl. Phys. B340 (1990) 281.

[33] E. Witten, Surveys in Diff. Geom. 1 (1991) 243.

[34] A.M. Polyakov, Gauge Fields and Strings, Harwood, New York, 1987.

[35] E. Witten, Nucl. Phys. B323 (1989) 113.

[36] J. Rabin, Nucl. Phys. B201 (1982) 315. 